\title{
Effectiveness, Safety and Tolerability of Gatifloxacin, a New 8- Methoxyfluoroquinolone, in the Treatment of Outpatients With Community- Acquired Pneumonia: A Brazilian Study
}

\author{
Suelene Aires Franca and \\ Carlos Roberto Ribeiro Carvalho
}

\author{
Pulmonary Division, Clinical Hospital of Medicine \\ School of São Paulo University, São Paulo, SP; \\ Intensive Care Unit - Clinical Hospital of Medicine \\ School of Federal University of Goiás, GO, Brazil
}

\begin{abstract}
Objectives: Evaluation of the effectiveness, safety and tolerability of gatifloxacin in the treatment of outpatients with community-acquired pneumonia (CAP). Study Design: A prospective, multicenter, non-comparative clinical study carried out in Brazil. Voluntary, unpaid physician participation contributed to an unbiased study design. Patients: Adult outpatients with clinical diagnosis of CAP. Regimen: Gatifloxacin, $400 \mathrm{mg}$ PO once daily for 7 to 14 days. Study Procedures: Initial clinical assessment, at the first day of gatifloxacin therapy; final evaluation after 7 to 14 days of treatment. Results: According to the physicians' assessments $97.3 \%$ of patients were cured or improved after gatifloxacin treatment. The incidence of adverse events was low and the most commonly reported events were nausea and dyspepsia. Conclusions: Gatifloxacin, $400 \mathrm{mg}$ PO once daily for 7 to 14 days, is effective and safe in the treatment of patients with CAP.

Key Words: Gatifloxacin, community-acquired pneunonia, Brazilian study.
\end{abstract}

Community-acquired pneumonia (CAP) is defined as a lower respiratory tract infection occurring in the community or within the first 48 hours after hospitalization. It remains one of the most common causes of infection-related morbidity-mortality, despite advances in diagnostic tests, antimicrobial therapy and specific vaccination.

During 1999, 969,752 patients diagnosed with pneumonia were admitted in hospitals pertaining to the "Sistema Único de Saúde - SUS" (Government Health Care System), in which $80 \%$ of population is attended. Considering the worldwide incidence of 12 CAP cases per 1,000 adult inhabitants, we can estimate an

Received on 22 March 2002; revised 16 July 2002.

Address for correspondence: Dr. Carlos Roberto Ribeiro Carvalho. Disciplina de Pneumologia - Hospital das Clínicas - FMUSP, $7^{\circ}$ Andar-sala 7079 - Ala E - Instituto Central. Av. Dr. Enéas de Carvalho Aguiar, 255 - Cerqueira César - São Paulo (SP), Brazil, Zip code: 05403-900.

The Brazilian Journal of Infectious Diseases 2002;6(4):157-163 (C) 2002 by The Brazilian Journal of Infectious Diseases and Contexto Publishing. All rights reserved. $1413-8670$ incidence of $1,920,000$ cases per year. Based on this consideration more than $50 \%$ of affected Brazilians are being treated in hospitals, in contrast with the data received from other countries, where $80 \%$ of CAP are treated in ambulatory facilities [1].

According to SUS data, in Brazil, pneumonia is considered the first cause of death in respiratory diseases and, except for external causes, it is classified in fourth place in general mortality among adults [1]. These data reflect American statistics, classifying CAP as the sixth most important cause of general mortality $[2,3]$.

In recent years, both the epidemiology and the treatment of CAP have changed, based on the recognition of new or previously non-identified pathogens, on the availability of more effective antibiotics and on the evolution of bacterial resistance mechanisms.

Organizations such as the American Thoracic Society (ATS) [2,3] and the Infectious Diseases Society of America (IDSA) [4,5] have proposed algorithms for empirical treatment of CAP. These are based on an assessment of the subjects (age, comorbidities), disease 
severity (related to the place indicated for the initial treatment: ambulatorial, hospital or intensive care unit) and pathogens known to be frequently associated with CAP in each specific condition. However, besides the careful assessment of these factors, the use of an antimicrobial agent requires knowledge about the activity of the antimicrobial agent against the presumed or known pathogen and its potential to induce bacterial resistance, besides safety and cost-effectiveness considerations [6].

Streptococcus pneumoniae, Haemophilus influenzae, Moraxella catarrhalis, Mycoplasma pneumoniae, Chlamydia pneumoniae and Legionella pneumophila remain themostcommon causative pathogens associated with CAP [7]. Though they are known to be relatively common organisms, increasing antimicrobial resistance of some of these pathogens to standard therapeutic regimens used in the treatment of PAC is a worldwide concern, due to the high prevalence rates of this disease.

$S$. pneumoniae remains the most important pathogen responsible for respiratory infections. Although resistance to penicillin does not represent a therapeutic problem in this condition (most resistant strains are susceptible to the therapeutic concentrations obtained in the plasma and pulmonary tissue), the choice of an antibiotic with low or no potential to induce resistance is critical in CAP treatment, to minimize the development of pneumococci highly resistant to penicillin $[6,8,9]$.

Gatifloxacin is a new advanced generation 8methoxifluoroquinolone that has an expanded spectrum of activity compared with previous generation quinolones $[10,11]$. It is active against gram-positive pathogens, including penicillin-resistant strains of $S$. pneumoniae and methicillin-susceptible $S$. aureus; gram-negative microorganisms, including $H$. influenzae and M. catarrhalis; atypical pathogens, such as Mycoplasma, Legionella and Chlamydia sp. [12-16]. When given by oral route, its bioavailability is $96 \%$ [17].

The efficacy of gatifloxacin in CAP treatment has been shown in randomized, double-blind (phase III) clinical trials, compared to levofloxacin [18], clarithromycin [19] and ceftriaxone \pm erythromycin [20].
Further studies are needed to confirm the placement of a new drug after its approval, in order to evaluate its effectiveness and the incidence of serious adverse events when it is prescribed to large populations, in surveillance programs that do not follow protocol procedures required for randomized clinical trials [21].

This prospective, multicenter, non-comparative clinical trial, conducted in Brazil, assessed the effectiveness, safety and tolerability of gatifloxacin in the treatment of adult outpatients with CAP.

\section{Materials and Methods}

We made a large-scale, community-based assessment of the effectiveness, safety and tolerability of gatifloxacin for the treatment of patients with CAP examined by primary care physicians or community specialists. Five hundred and four physicians, across Brazil, participated in the study over a 6-month period from March to October, 2000. The participation of physicians in this study was entirely voluntary.

Study Population. Inclusion criteria: adult patients ( $>$ 18 years old) with clinical diagnosis of CAP, for whom oral therapy was indicated and who agreed to participate in this study.

Exclusion criteria: history of hypersensitivity reaction to fluoroquinolones; pregnancy or breast-feeding; inability to use an effective contraception method during the study; diagnosis of asthma or cystic fibrosis; nonbacterial respiratory tract infections; gastrointestinal disturbances affecting drug absorption.

Definition. CAP was diagnosed by pulmonary auscultatory findings, such as rales and/or egophony, associated with two or more of the following signs and symptoms: fever $\left(>38.0^{\circ} \mathrm{C}\right)$, chest pain, cough, sputum production, chills or general discomfort [4].

Study Protocol. Each patient underwent a clinical examination on initiation of treatment and 7 to 14 days after therapy began. The evaluation of each patient was recorded in a case report form designed 
for this study, completed by the responsible physician. The initial assessment included, besides the clinical diagnosis of CAP, based on the definition given above, demographics (age, sex), smoking history, history of previous respiratory tract infections (specimen and number within last 12 months before the study), exposure to antibiotics within 3 months before the study, and use of concomitant medications. At the time of the follow-up visit, patients were carefully questioned about any adverse events or reactions.

Patients were prescribed gatifloxacin $400 \mathrm{mg}$ PO once daily for 7 to 14 days, at the discretion of the investigator. The drug could be administered without regard to meals.

Effectiveness assessment. Assessment of treatment effectiveness, defined as clinical response rate, was classified as:

(1) Cured: All symptoms of infection resolved and no additional antibiotic therapy was required;

(2) Improved: Evident reduction of infection symptoms, but incomplete resolution of some symptoms; no additional antibiotic therapy was required.

(3) Relapse: After initial improvement, the symptoms worsened during therapy, requiring maintenance of antibiotic therapy.

(4) Therapeutic failure: Poor improvement of the symptoms during antibiotic therapy.

(5) Unable to determine: Lost to follow-up, early termination of therapy or unable to assess due to interference by other conditions.

Adverse events assessment. Evaluation of adverse effects to the therapy was assessed throughout the medical report of the adverse event, including its intensity (mild, moderate, serious, extremely serious), relationship to the study drug (certain, probably, possibly, not likely), action taken regarding the study drug (none, dose reduction, interruption, discontinuation, dose increase) and the need for treatment.
Statistics. As the study was an open, non-comparative assessment of the effectiveness and the safety of gatifloxacin, only descriptive statistics were used for analysis of the results.

\section{Results}

Characteristics of the patients. A total of 1,501 patients were assessed, in different regions of the country, from March to October 2000. Demographic and clinical data are shown in Table 1.

Effectiveness. The physicians' evaluations of the responses to treatment showed that of the 1,501 patients, 1,460 (97.3\%) were considered clinically cured $(1,198)$ or improved $(262)$ after receiving gatifloxacin therapy. Only $14(0.9 \%)$ of the subjects were considered to have therapeutic failure (Table 2).

Safety and tolerability. Among the 1,501 assessed patients, $128(8.5 \%)$ developed at least one adverse event.

Of a total of 158 adverse events, 109 were reported as probably, possibly or not likely related to gatifloxacin (Table 3).

One hundred and five adverse events (85.5\%) were considered of mild or moderate intensity. Of the 20 adverse events considered as serious or extremely serious, those related to gatifloxacin therapy were gastrointestinal disturbances (nausea, vomiting, diarrhea, epigastric pain) and disturbances of the central nervous system (vertigo, chills, headache).

The most commonly reported adverse events in 128 patients were nausea (14\%), dyspepsia (11\%), diarrhea (7\%), vertigo (6\%) and headache (4\%). All of these are typical adverse events seen in patients on antimicrobial therapy.

\section{Discussion}

Community-acquired pneumonia is one of the most common types of respiratory tract infections. In USA 
Table 1. Demographics and clinical characteristics of 1,501 outpatients clinically diagnosed with CAP

\begin{tabular}{|c|c|}
\hline Characteristic & \\
\hline Age, years* & $49 \pm 19$ \\
\hline \multicolumn{2}{|l|}{ Sex, $N^{o \cdot}(\%)$} \\
\hline Male & 718 (47.9) \\
\hline Female & $782(52.1)$ \\
\hline Not informed & $1(0.01)$ \\
\hline \multicolumn{2}{|l|}{ Smoker, $N^{\circ} \cdot(\%)$} \\
\hline Yes & $330(22.1)$ \\
\hline No & $1,166(77.9)$ \\
\hline Never smoked & $688(73.5)$ \\
\hline Ex-smoker & $248(26.5)$ \\
\hline Not informed & $230(19.7)$ \\
\hline Not informed & $5(0.3)$ \\
\hline \multicolumn{2}{|c|}{ Signs and Symptoms, $\mathrm{N}^{\circ}(\%) \dagger$} \\
\hline Fever & $1,324(89.40)$ \\
\hline Chest Pain & $1,064(71.84)$ \\
\hline Cough & $1,393(94.06)$ \\
\hline Sputum Production & $1,172(79.14)$ \\
\hline Chills or malaise & $1,181(79.74)$ \\
\hline
\end{tabular}

Table 2. Effectiveness of gatifloxacin $400 \mathrm{mg}$ PO once daily for treatment of 1,501 outpatients clinically diagnosed with CAP

\begin{tabular}{lc}
\hline Clinical evolution & $\mathbf{N}^{\mathbf{0}} \mathbf{( \% )}$ \\
\hline Cured & $1,198(79.8)$ \\
Improved & $262(17.5)$ \\
Relapse & $4(0.3)$ \\
Failure & $14(0.9)$ \\
Unable to evaluate & $23(1.5)$ \\
Total & $1,501(100)$ \\
\hline
\end{tabular}


Table 3. Characteristics of 158 adverse events occurring in 128 outpatients clinically diagnosed with CAP

\begin{tabular}{lc}
\hline Intensity, No. (\%) & \\
Mild & $75(47.5)$ \\
Moderate & $60(38.0)$ \\
Serious & $16(10.1)$ \\
Extremely serious & $4(2.5)$ \\
Not informed & $3(1.9)$ \\
Relationship to the study drug, No. (\%) & \\
Certain & $44(27.8)$ \\
Probably & $64(40.5)$ \\
Possibly & $38(24.1)$ \\
Not likely & $7(4.4)$ \\
Not informed & $5(3.2)$ \\
Action taken related to the drug, No. $(\%)$ & \\
None & $126(79.7)$ \\
Dose Reduction & $2(1.3)$ \\
Interruption & $14(8.9)$ \\
Discontinuation & $9(5.7)$ \\
Not informed & $7(4.4)$ \\
Treatment required, No. (\%) & \\
No & $106(67.1)$ \\
Yes & $46(29.1)$ \\
Not informed & $6(3.8)$ \\
\hline
\end{tabular}

it remains as the most common cause of infectionrelated mortality $[2,3]$.

Community-acquired pneumonia (CAP) treatment is complex, due to a broad spectrum of potential etiological agents, the development of resistance among these pathogens and the difficulty to make a microbiological diagnosis, even with the use of sophisticated diagnostic tests. Due to these factors, antimicrobial therapy should be effective against common and atypical etiological agents and it is expected that new antibiotics will have high activity and minimal cross-resistance with other drugs $[2,3,8,9]$.

Antimicrobial therapy should be started as early as possible for CAP treatment, for this reason, and due to the limitations of our current diagnostic methods, it is usually empirical. The options for antimicrobial therapy are in continuous development. For initial empirical therapy in immunocompetent adult patients, with no indication for hospitalization, to date advanced generation macrolides are recommended; doxycycline, $\beta$-lactam/macrolide combination and new fluoroquinolones (antipneumococcal fluoroquinolones) are other options $[3,8,9]$.

The results observed in this study, with high cure or clinical improvement rates $(97.3 \%)$ shown by patients with CAP who received gatifloxacin, were similar to the results of phase III studies [18-20] and another recent phase IV study, conducted in Mexico, of 3,322 patients with CAP treated with gatifloxacin, that had a success rate of $95.8 \%$ [22]. 
The adverse events observed with antimicrobial agents should also be considered and the choice of a safe agent for the treatment of CAP is required. The results of this study, which evaluated 1,501 patients with CAP, showed that adverse events related to gatifloxacin therapy were typical and similar to those observed with other antimicrobial therapies, such as nausea, diarrhea and headache.

Our study has some limitations. First, the definition ofCAP that we used may be inadequate. We considered that possibility, but although we recommend that all patients with CAP should have a chest radiograph to establish the diagnosis and to determine if there are complications, in some outpatient settings this may be difficult or even impossible, depending on the time of the day and the availability of a radiology facility. Because this was a study of the effectiveness and safety of gatifloxacin in the "real world", outside the restricted environment of phase III clinical trials, we decided to use a clinical definition of CAP, which consisted of newly acquired respiratory or systemic symptoms or signals of infection accompanied by auscultatory findings of abnormal breath sounds and/or crackles. Although a diagnosis of pneumonia should be considered in any patient who has this clinical picture, these criteria do not have optimal sensitivity and specificity. Finally, we were unable to ascertain the number of patients that complied with the inclusion criteria during the study period but could not be fully evaluated. Actually, some of these (unevaluated) patients may have had different outcomes, but due to the sample size that we completely evaluated, we consider the results representative for the general population.

Based on our data and on a review of the literature [18-20,22], gatifloxacin is an important therapeutic option for CAP, due to its efficacy, effectiveness and the absence of known serious adverse events usually associated with other fluoroquinolones [23].

\section{Conclusion}

Oral gatifloxacin administered at a dosage of 400 mg PO once daily for 7 to 14 days was found to be effective and safe in this open, multicenter, noncomparative clinical trial of 1,501 outpatients clinically diagnosed with community-acquired pneumonia. Cure or clinical improvement occurred in $97.3 \%$ of the patients. The rate and seriousness of adverse events were low.

\section{Acknowledgment:}

The authors thank Bristol-Myers Squibb - Brazil for technical support during this study.

\section{References}

1. Sociedade Brasileira de Pneumologia e Tisiologia. Consenso brasileiro de pneumonias em indivíduos adultos imunocompetentes. Jornal de Pneumologia 2001;27:S3-S21.

2. Niederman M.S., Bass J.B. Jr., Campbell G.D., et al. American Thoracic Society Medical Section of the American Lung Association. Guidelines for the initial management of adults with community-acquired pneumonia: diagnosis, assessment of severity and initial antimicrobial therapy. Am Rev Respir Dis 1993;148:1418-26.

3. Niederman M.S., Mandell L.A., Anzueto A., et al. American Thoracic Society. Guidelines for the management of adults with community-acquired pneumonia: diagnosis, assessment of severity, antimicrobial therapy, and prevention. Am J Respir Crit Care Med 2001;163:1730-54.

4. Barlett J.G., Breiman R.F., Mandell L.A., et al. Communityacquired pneumonia in adults: guidelines for management. Clin Infect Dis 1998;26:811-38.

5. Barlett J.G., Dowell S.F., Mandell L.A., et al. Practice guidelines for the management of community-acquired pneumonia in adults. Clin Infect Dis 2000;31:383-421.

6. Cunha B.A. Community-acquired pneumonia: diagnostic and therapeutic approach. Med Clin North Am 2001;85:43-77.

7. Marrie T.J. Community-acquired pneumonia: epidemiology, etiology, and treatment. Infect Dis Clin North Am 1998; 12:723-40.

8. Bernstein J.M. Treatment of community-acquired pneumonia - IDSA guidelines. Chest 1999;115:9S-13S.

9. Campbell G.D. Commentary on the 1993 American Thoracic Society guidelines for the treatment of communityacquired pneumonia. Chest 1999;115:14S-18S.

10. Fung-Tome J., Minassian B., Kolek B., et al. In vitro antibacterial spectrum of a new broad-spectrum 8methoxy fluoroquinolone, gatifloxacin. J Antimicrob Chemother 2000;45:437-46. 
11. Hershberger E., Rybak M.J. Activities of trovafloxacin, gatifloxacin, clinafloxacin, sparfloxacin, levofloxacin, and ciprofloxacin against penicillin-resistent Streptococcus pneumoniae in an in vitro infection model. Antimicrob Agents Chemother 2000;44:598-601.

12. Blondeau J.M. Expanded activity and utility of the new fluoroquinolones: a review. Clin Ther 1999;21:3-40.

13. Jones R.N, Biedenbach D.J., Erwin M.E., et al. Activity of gatifloxacin against Haemophilus influenzae and Moraxella catarrhalis, including susceptibility test development, e-test comparisons, and quality control guidelines for $H$. influenzae. J Clin Microbiol 1999;37:1999-2002.

14. Croco M.A.T., Biedenbach D.J., Pfaller M.A., et al. Invitro activities of gatifloxacin, sparfloxacin and trovafloxacin against 103 strains of Legionella spp. J Antimicrob Chemother 1998;48:672-8.

15. Diekema D.J., Jones R.N., Roiston K.V. Antimicrobial activity of gatifloxacin compared to seven other compounds tested against gram-positive organisms isolated at 10 cancer-treatment centers. Diagn Microbiol Infect Dis 1999;34:37-43.

16. Odland B.A., Jones R.N., Verhoef J., et al. Antimicrobial activity of gatifloxacin (AM-1155, CG5501), and four other fluoroquinolones tested against 2,284 recent clinical strains of Streptococcus pneumoniae from Europe, Latin America, Canada, and the United States. Diagn Microbiol Infect Dis 1999;34:315-20.

17. LaCreta F.P., Kaul S., Kollia G.D., et al. Interchangeability of 400-mg intravenous and oral gatifloxacin in healthy adults. Pharmacotherapy 2000;20:59S-66S.

18. Sullivan J.G., McElroy A.D., Honsinger R.W., et al. Treating community-acquired pneumonia with oncedaily gatifloxacin vs once-daily levofloxacin. J Respir Dis 1999;20:49S-59S.

19. Ramirez J.A., Nguyen T., Tellier G., et al. Treating community-acquired pneumonia with once-daily gatifloxacin vs twice-daily clarithromycin. J Respir Dis 1999;20:40S-8S.

20. Fogarty C., Dowell M.E., Ellison W.T., et al. Treating community-acquired pneumonia in hospitalized patients: gatifloxacin vs ceftriaxone/clarithromycin. J Respir Dis 1999;20:60S-9S.

21. Hulley S.B., Cummings S.R., Browner W.S., et al. Designing clinical research, $2^{\text {nd }}$ ed. Lippincott Williams \& Wilkins, 2001.

22. Casillas J.L., Rico G., Rodríguez-Parga D., et al. Multicenter evaluation of the efficacy and safety of gatifloxacin in Mexican adult outpatients with respiratory tract infections. Advances in therapy 2000;17:263-71.

23. Breen J., Skuba K., Grasela D. Safety and tolerability of gatifloxacin, an advanced-generation, 8-methoxy fluoroquinolone. J Respir Dis 1999;20:70S-6S. 